
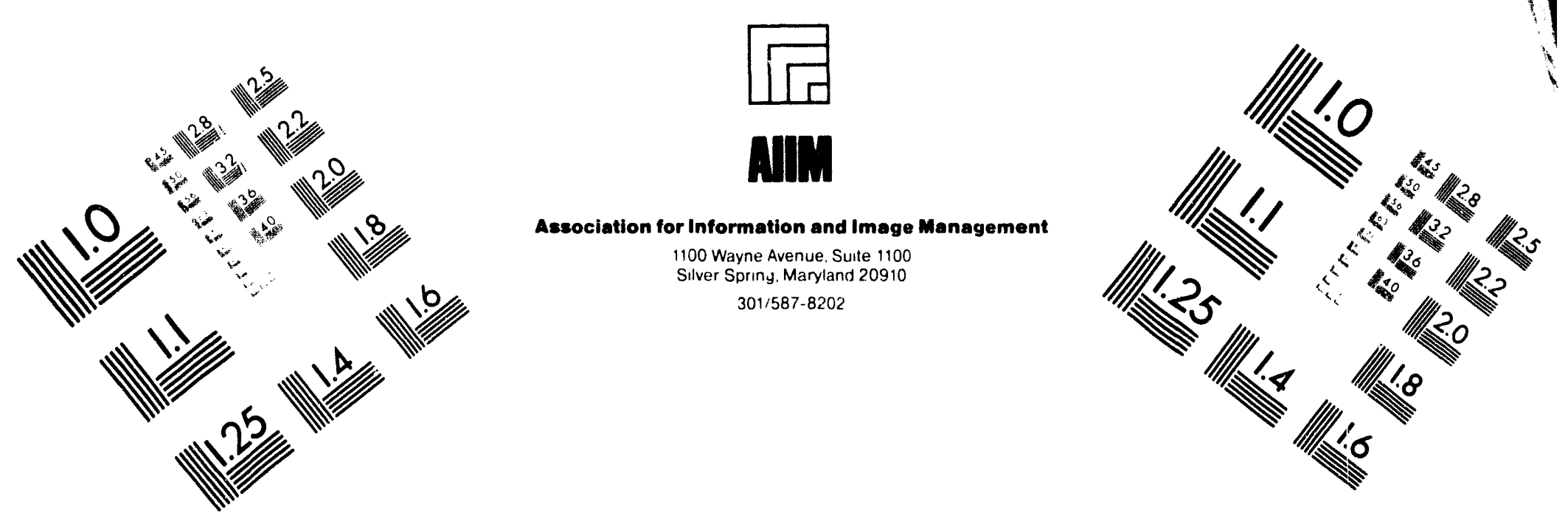

\title{
Centimeter
}

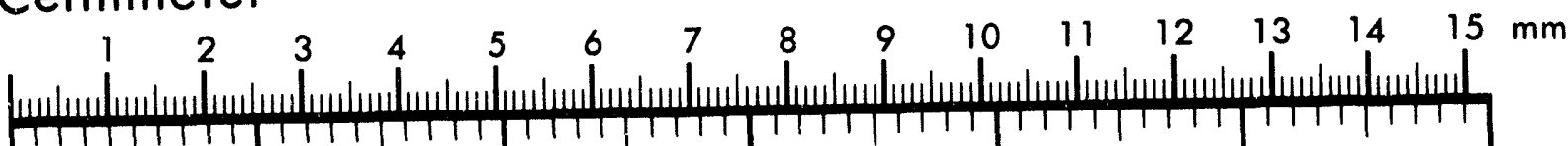

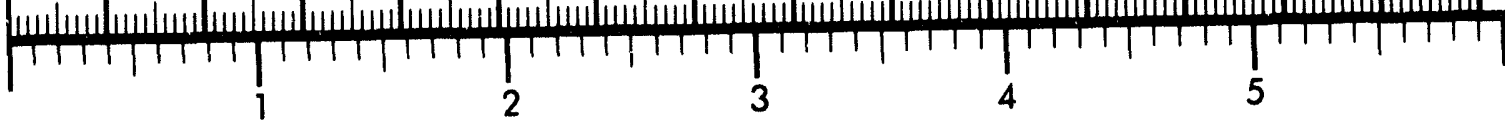
Inches
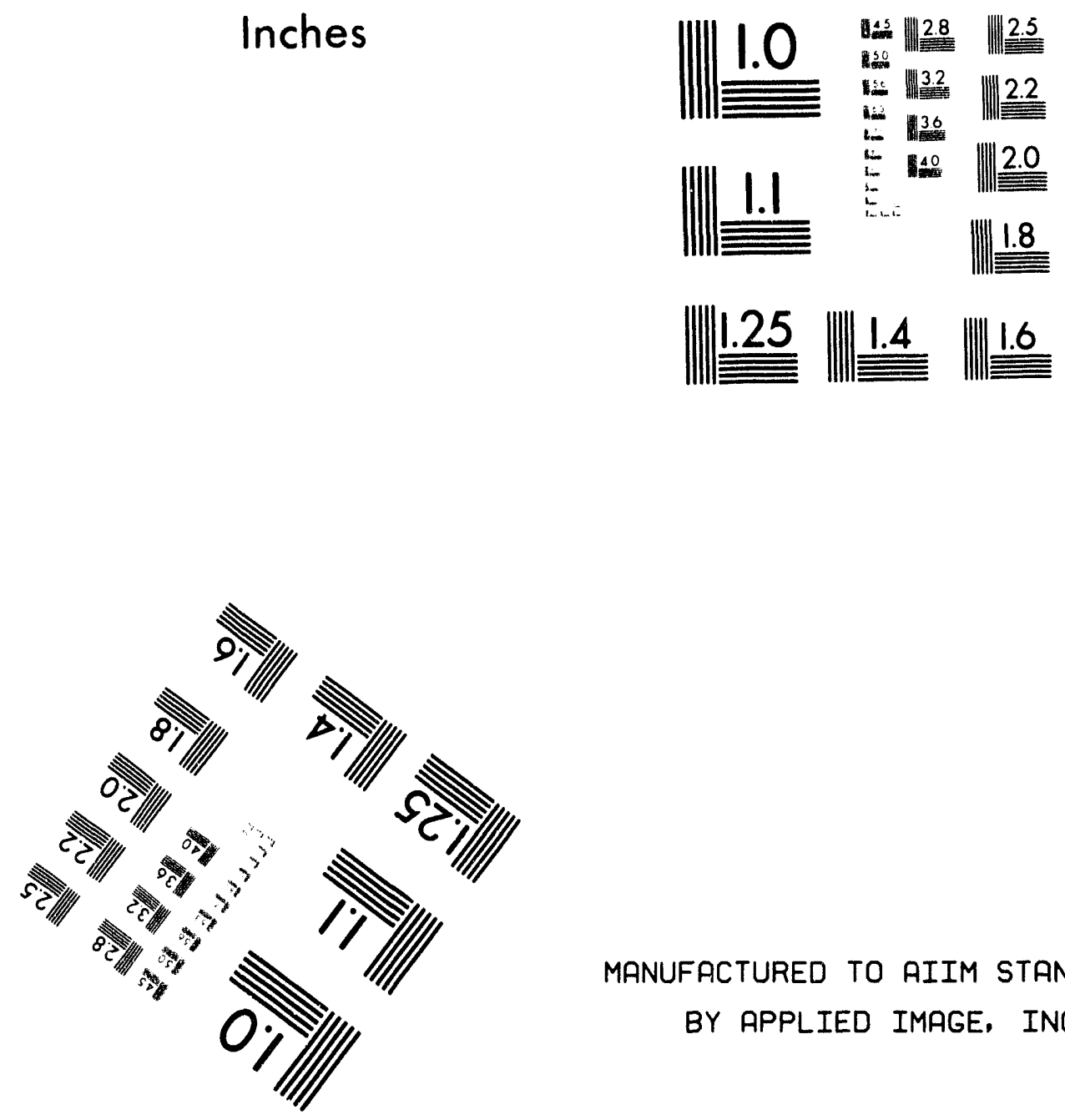

MANUFACTURED TO AIIM STANDARDS

BY APPLIED IMAGE, INC.

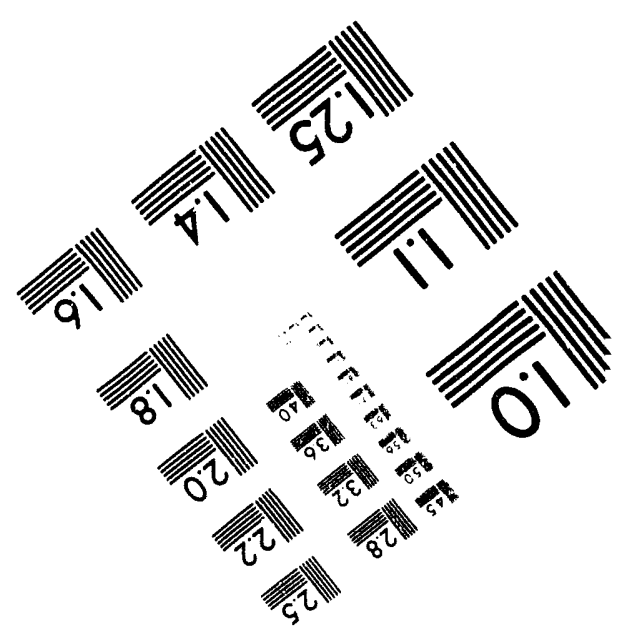



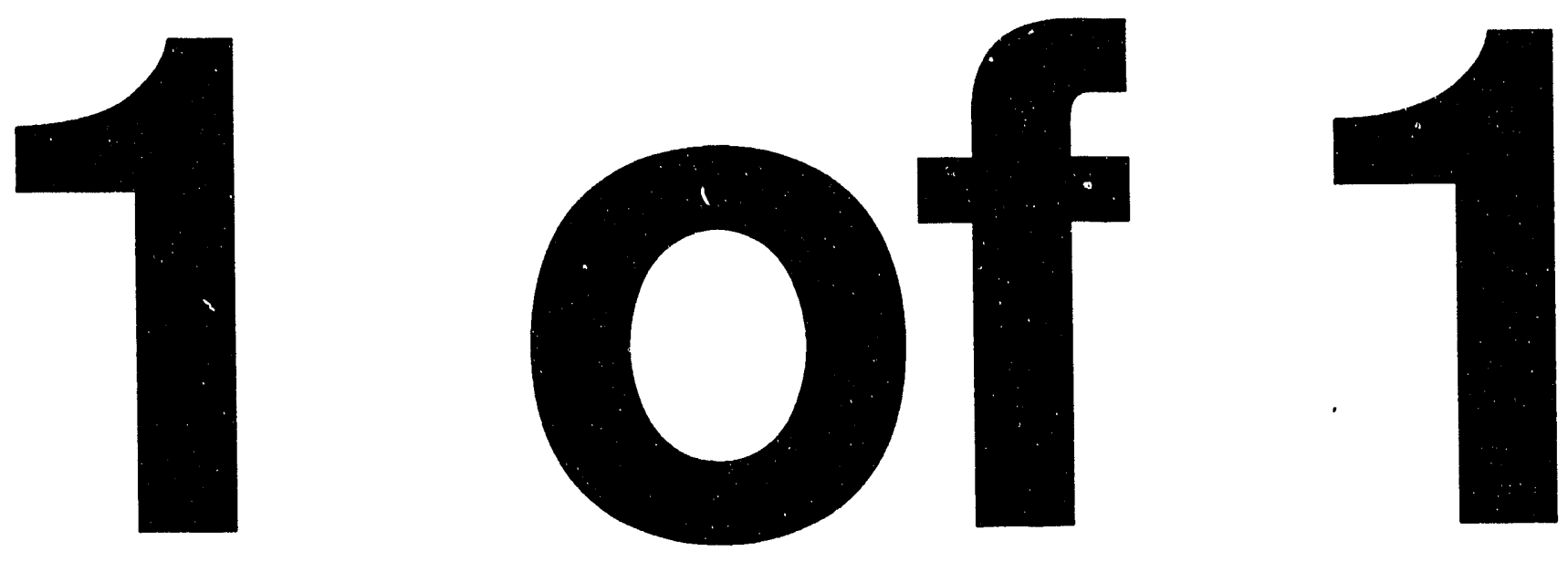


\title{
FEEST: A Method For Tabulating Equation of State and Opacity Tables
}

\author{
Richard H. White \\ University of California \\ Lawrence Livermore National Laboratory \\ Livermore, CA 94550
}

March 1993

- This is an informal report intended primarily for internal or limited external distribution. The opinions and conclusions stated are those of the author and may or may not be those of the Laboratory.

Work performed under the auspices of the U.S. Department of Energy by the

- Lawrence Livermore National Laboratory under Contract W-7405-Eng-48.

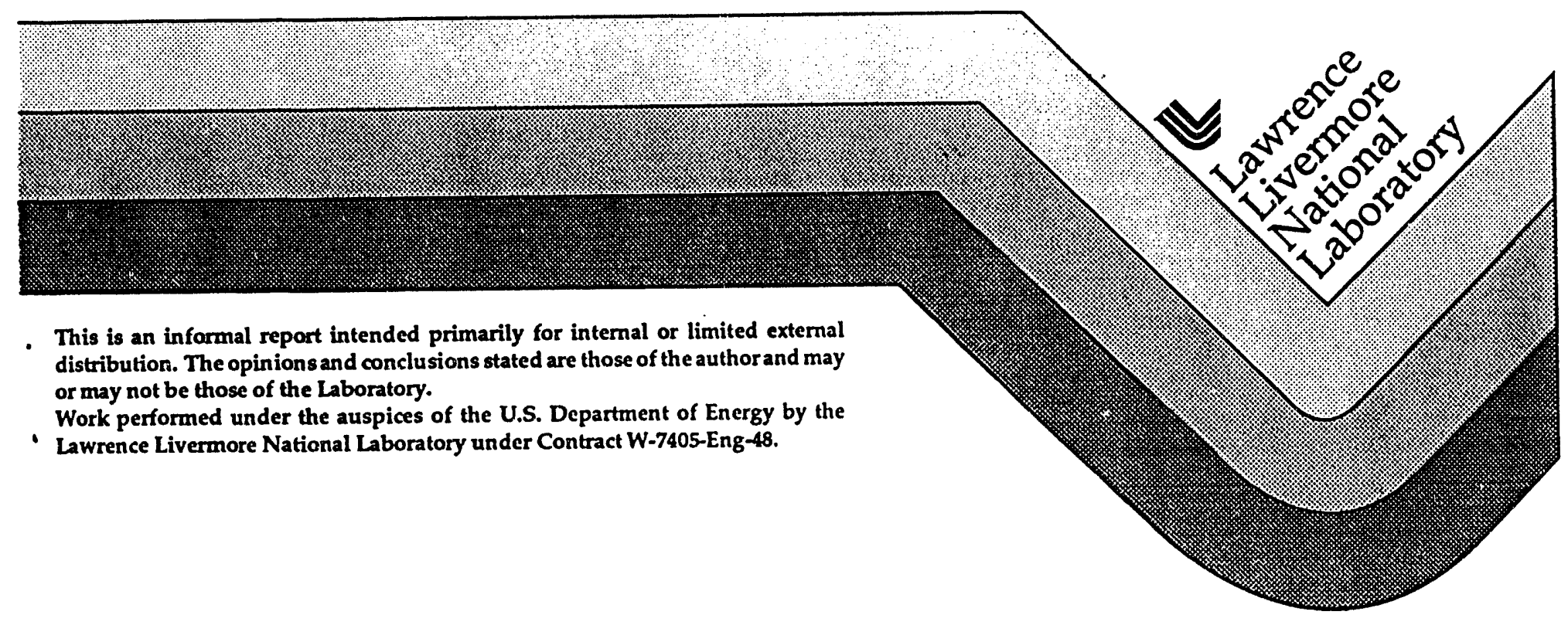


This document was prepared as an acooount of work sponsored by an agency of the United States Government. Neither the United States Government nor the University of California nor any of their employees, makes any warranty, express or implied, or assumes any legal liability or responsibility for the accuracy, completeness, or usefulness of any information, apparatus, product, or process disclosed, or represents that its use would not infringe privately own rights. Reference herein to any spedfic commercinl products, process, or service by trade name, trademar'k, manufacturer, or otherwise, does not necessarily constitute or imply its endorsement, recommendation, or favoring by the United States Government or the University of California. The views and opinions of authors expressed herein do not necessarlly state or reflect those of the United States Government or the University of California, and ahall not be used for advertising or product endorsement purposes.

This report has been reproduced directly from the best available copy.

Available to DOE and DOE contractors from the Office of Scientific and Technical Information P.O. Box 62, Oak Ridge, IN 37831

Prices available from (615) 576-8401, FTS 626-8401

Available to the public from the National Technical Information Service

U.S. Department of Commerce 5285 Port Royal Rd. Springfield, VA 22161 


\section{FEEST: A MIETHOD FOR TABULATING \\ EQUATION OF STATE AND OPACITY TABLES}

R. H. White

\section{FOREWARD}

This report is an edited version of a memorandum which was issued circa 1973. The techniques described are limited by the computer systems available in the early 1970's.

The essential ideas in the FEEST system (described in this report) remain valid today. In particular, the derivation of equation of state data from tabulated thermodynamic potentials would be an important improvement over the bi-quadratic-Lagrange pressure-energy tabulations which have been used at LLNL since the early 1960's.

Present day ?omputing capacity (e.g., with the CRAY machines) encourages more sophisticated techniques than are described in this report. It is probably now feasible to construct an equation-of-state tabulation and interpolation scheme similar to FEEST which is (a) thermodynamically consistent, (b) continuous everywhere (including continuity of specific heats, bulk modulus and other derivatives of the thermodynamic functions), and (c) competitive in speed with the bi-quadratic-Lagrange tabulations.

\section{INTRODUCTION}

The tabulation of equations of state presently used by codes which carry temperature and density as independent variables, provides separate tables for pressure $(P)$ and specific internal energy $(\varepsilon)$ as functions of the temperature $(T)$ and density $(\rho)$. Both $\mathrm{P}$ and $\varepsilon$ are determined by bi-quadratic interpolation in tables of $\mathrm{P}$ and $\varepsilon$ values arranged on a rectangular grid in the $T-\rho$ plane. ${ }^{1,2}$ The system was designed for use on the 709-7094 generation of computers and has been used, essentially unmodified, for approximately twenty years.

We have long recognized that this "Bi-quadratic Tabulation" system has several dra wbacks:

1. The interpolation produces values of $P$ and $\varepsilon$ which are not consistent with the $2^{\text {nd }}$ law of thermodynamics. The $2^{\text {nd }}$ law requires $T(\partial P / \partial T)_{v}=P+(\partial \varepsilon / \partial v)_{T}$ (where $\left.v=1 / 0\right)$. Since the fitted data are, presumably, consistent with the $2^{\text {nd }}$ law, the discrepancy is not 
usually catastrophic. Dana Warren has investigated this problem and has modified the fitting procedure for the bi-quadratic tabulation to alleviate the discrepancy. 3,4 Nevertheless, it is not possible to uniquely define an entropy function in the bi-quadratic tabulation scheme.

2. Boxes in the tables are the rectangles bounded by the "grid lines" $T=T_{j}(j=1, J)$ and $\rho=\rho_{k}(k=1, K)$. For each box, 9 interpolation coefficients must be stored in memory. To obtain good fits in regions where $P$ and $\varepsilon$ vary faster than quadratically with $T$ or $\rho$, small boxes have to be introduced in other regions. The table size is determined in those regions which are most difficult to fit.

3. Derivatives of the thermodynamic functions are not necessarily continuous across table grid lines. It is possible when fitting the data to place constraints on the interpolation coefficients which will force the continuity of derivatives across grid lines but this places severe restrictions on the coefficients and leads to small boxes - - i.e., large tables. The difficulty could be alleviated by including more terms in the interpolation e.g., by using bi-cubic interpolation (but the number of stored coefficients then must be, approximately, half again as large).

In this paper, we describe the Free Energy Equations of State Tabulation (FEEST) scheme which is intended to replace the presently used Bi-Quadratic Tabulation. The scheme described has the following advantages:

1. P and $\varepsilon$ values are necessarily consistent with the $2^{\text {nd }}$ law and hence one can define the entropy and any other thermodynamic function.

2. The table is divided into regions which may be any shape that can be made from rectangles bounded by grid lines $T=T_{j}, \rho=\rho_{k}$ (thus admitting "T" shaped and " $L$ " shaped regions as well as such bizarre shapes as "square doughnuts" or "stair steps" which cut across the region of interest in the $\mathrm{T}-\rho$ plane). Furthermore, regions of the $\mathrm{T}-\rho$ plane can be excluded from the tables, thus making it unnecessary to fit regions where data is unavailable and/or which will not be reached during the course of calculations.

3. The fitting scheme has built-in features for "matching" the values of $P, \varepsilon$, and their first derivatives at region boundaries and the method can be extended to match the values of any other thermodynamic function of state at such boundaries.

The Bi-quadratic Tabulation, now used, treats opacities $(\kappa)$ in the same way as $P$ and $\varepsilon$ expect that $(1 / T)$ replaces $T$ as one of the interpolation variables. Since $k$ tnay vary inversely with a high ( 3 to 8 ) power of $\mathrm{T}$ and since bi-quadratic interpolation in $(1 / \mathrm{T})$ includes terms only up to $\mathrm{T}^{-2}$, opacity tables have been large and difficult to produce. 
The FEEST system circumvents this difficulty by fitting $\ln (\kappa)$ with a function biquadratic in $\ln (T)$ and $\ln (\rho)$. Thus in a region where $\kappa$ is proportional to $T^{-n}$ (n constant), the FEEST fits are to a function linear in the independent variable $\ln (T)$; the terms quadratic in $\ln (\mathrm{T})$ then, in effect, allow for $\mathrm{n}$ to be a (slowly varying) function of $\mathrm{T}$ and of $\rho: n=n_{0}(\rho)+n_{1}(\rho) \ln T$. Table sizes are from half to two thirds those presently used and the work required to produce a good fit has been considerably reduced.

\section{THE HELM HOLTZ FREE ENERGY AS A TABULATED FUNCTION}

The $2^{\text {nd }}$ law requires that there exist an entropy function $S(T, p)$ which satisfies the Pfaffian differential relation

$$
\mathrm{TdS}=\mathrm{Pdv}+\mathrm{d} \varepsilon
$$

When using $T$ and $\rho$ (or $v$ ) as independent variables, it is convenient to work with the Helmholtz Free Energy:

$$
F=\varepsilon-T S
$$

then

$$
\mathrm{dF}=\mathrm{d} \varepsilon-(\mathrm{TdS}+\mathrm{SdT})
$$

and by virtue of Eg. (1)

$$
\mathrm{dF}=-\mathrm{Pdv}-\mathrm{SdT}
$$

so that

$$
S=-\left(\frac{\partial F}{\partial T}\right)_{v} \equiv-F_{T}
$$

and

$$
P=-\left(\frac{\partial F}{\partial v}\right)_{T} \equiv-F_{V}
$$

(we shall, henceforth, use subscripts $\mathrm{T}$ and $\mathrm{v}($ or $\rho$ ) to denote partial differentiation with respect to the one of these variables occurring in the subscript while the other is held fixed).

From $\mathrm{F}$, we can generate all the thermodynamic functions and their derivatives and they will automatically satisfy the $2^{\text {nd }}$ law because the definition of $F$ predicates the existence of an entropy function [see Eq. (2) ]. 
For example, from Eq. (2), we have

$$
\varepsilon=F+T S
$$

and by substitution from Eq. (4), we obtain

$$
\varepsilon=\mathrm{F}-\mathrm{TF}_{\mathrm{T}}
$$

and by differentiation of Eq. (6)

$$
\begin{aligned}
& C_{v}=\varepsilon_{T}=-T_{T T} \\
& \varepsilon_{v}=F_{v}-T F_{T v}
\end{aligned}
$$

The relation by which Dana Warren has called the "T relation" is just Eq. (7) with -P substituted for $F_{v}$ [see Eq. (5) ], i.e.,

$$
\varepsilon_{v}=-P+T P_{T}
$$

As noted above, we can generate any of the thermodynamic functions by taking appropriate combinations of $\mathrm{F}$ and its derivatives. Therefore the FEEST system replaces the tabulations of $P$ and $\varepsilon$ now used, by a single tabulation of $F$ as a function of $T$ and $\rho$. The thermodynamic functions obtained from $\mathrm{F}$ then automatically satisfy the $2^{\text {nd }}$ law.

\section{MODIFIED TABLE LOOK-UP}

\section{Presently Used Scheme}

The bi-quadratic tabulation requires that the table be divided into rectangular boxes by grid lines $T=$ constant, $\rho=$ constant. Each table is assigned a number $(N)$. (For each material used in a calculation, there are three tables: $P, \varepsilon, \kappa . N$ indexes not only the material needed but also the particular table.) The grid lines are indexed by the arrays $\operatorname{ITES}(\mathrm{N})$ and $\operatorname{IRES}(\mathrm{N})$ as follows: $\operatorname{TES}[\operatorname{ITES}(\mathrm{N})]$ is $\mathrm{T}$ for the first $\mathrm{T}$-grid line in Table $N$, $\operatorname{TES}[\operatorname{ITES}(N)+1]$ is the second, etc. (see Fig. I). Thus a table box for table $N$ is bounded by the lines $\operatorname{TES}(j), \operatorname{TES}(j+1), \operatorname{RES}(k), \operatorname{RES}(k+1)$, where $\operatorname{ITES}(N) \leq j<\operatorname{ITES}(N+1)-1$ and $\operatorname{IRES}(N) \leq k<\operatorname{IRES}(N+1)-1$. To this box there is assigned an index $m$ which recovers the interpolation coefficients $\operatorname{AES}(\mathrm{m}), \mathrm{BES}(\mathrm{m})$, etc. 


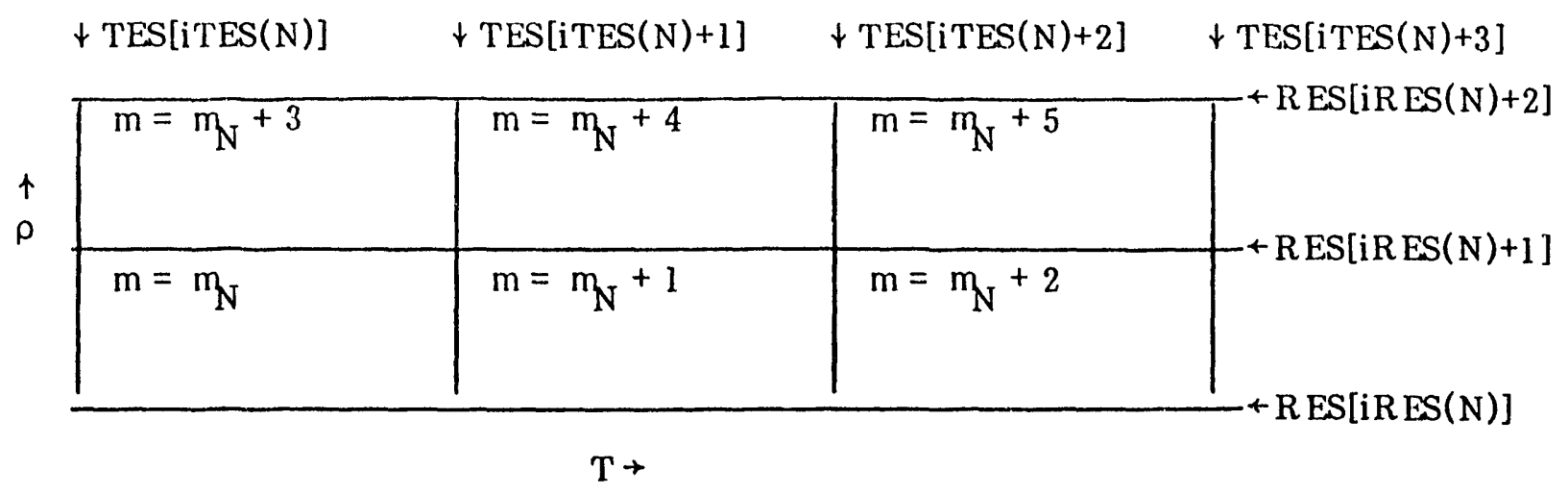

FIG. I. A $3 \times 5$ box table in the bi-quadratic tabulation scheme. Temperature and density grid line are stored in the arrays TES and RES, respectively. The indices " $m$ " reference boxes in the table. Each box has a corresponding set of interpolation coefficients, $\operatorname{AES}(m), \operatorname{BES}(m), .$.

\section{Proposed Modification}

The FEEST system adds to this nest of indices one more index: $M R E G(m) \equiv M$ which will identify the box with a "region", which comprises one or more boxes, and indexes the coefficients in the expansion of F with M (see Fig. II). The time spent in table look-1.p with this modified scheme is essentially the same as with the biquadratic scheme. There is just one additional indexing operation required and the index need be calculated only when an entry into the table is to a box different from that of the previous entry. [Note: In the FEEST System there are just two tables ( $F$ and $K$ ) per material.]

Furthermore, "dummy" region numbers (indicated e.g., by MREG being negative) can be assigned to parts of the $\mathrm{T}-\rho$ plane which are outside the table and hence the rather complicated search procedure now used for determining when one has left the table can be eliminated.

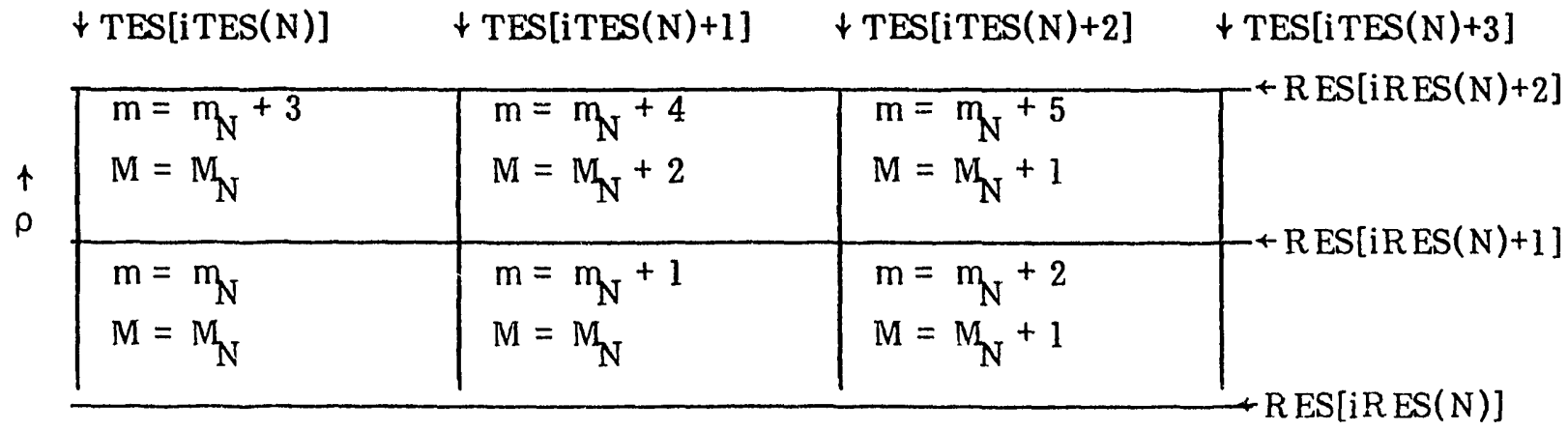

$$
\begin{aligned}
& \mathrm{T} \rightarrow
\end{aligned}
$$

FIG. II. A $3 \times 5$ box table (in the FEEST scher.e) which has been divided into 3 regions (bounded by the heavy lines). The indices " $\mathrm{m}$ " reference boxes; the indices " $M$ " reference regions. The map of box numbers into region numbers is the array $M R E G(m)$. In the Figure $\operatorname{MREG}\left(m_{N}\right) \equiv M_{N}, M R E G\left(m_{N}+1\right)=M_{N}, M R E G\left(m_{N}+2\right)=M_{N}+1$, etc. Each region has a corresponding set of interpolation coefficients [called $A(M, n)$ in Eq. (9) ]. 
THE FORMS OF THE TABULATED FUNCTIONS

\section{Free Energy}

The FEEST system represents the Helmholtz Free Energy by an expansion linear in a set of basis functions:

$$
F(T, \rho)=\sum_{n} A(M, n) \quad f_{n}(T, \rho)
$$

where the coefficients $A(M, n)$ are constant throughout region $M$ but may vary from one region to another and the "basis" functions $f_{n}(T, \rho)$ are the same for all regions and materials. A considerable amount of experimentation has led to the following choice of $\mathrm{f}_{\mathrm{n}}$ 's:

$$
\begin{aligned}
\mathrm{f}_{1} & =1 \\
\mathrm{f}_{2} & =\rho \\
\mathrm{f}_{3} & =\rho^{2} \\
\mathrm{f}_{4} & =\rho^{3} \\
\mathrm{f}_{5} & =\ln \rho \\
\mathrm{f}_{6} & =\mathrm{T} \ln \mathrm{T} \\
\mathrm{f}_{7} & =\rho \mathrm{T} \ln \mathrm{T} \\
\mathrm{f}_{8} & =\rho^{2} \mathrm{~T} \ln \mathrm{T} \\
\mathrm{f}_{9} & =\rho^{3} \mathrm{~T} \ln \mathrm{T} \\
\mathrm{f}_{10} & =\mathrm{T}^{2} \\
\mathrm{f}_{11} & =\rho \mathrm{T}^{2} \\
\mathrm{f}_{12} & =\rho^{2} \mathrm{~T}^{2} \\
\mathrm{f}_{13} & =\rho^{3} \mathrm{~T}^{2} \\
\mathrm{f}_{14} & =\mathrm{T}^{2} \ln \rho \\
\mathrm{f}_{15} & =\rho \mathrm{T} \\
\mathrm{f}_{16} & =\mathrm{T} \ln \rho
\end{aligned}
$$

With this choice of basis functions, the expression for F in Eq. (9) is conveniently summarized in the following table in which $\mathrm{f}_{\mathrm{n}}$ is the product of the variables at the heads of the column and row in which the corresponding coefficient $\left[A_{n} \equiv A(M, n)\right]$ appears. " $X$ " indicates positions where no corresponding terms appear in the expansion of $F$ in Eq. (9). 


\begin{tabular}{c|c|c|c|c|c|}
\multicolumn{1}{c}{1} & \multicolumn{1}{c}{$\rho$} & $\rho^{2}$ & $\rho^{3}$ & \multicolumn{1}{c}{$\ln \rho$} \\
\cline { 2 - 7 } & $A_{1}$ & $A_{2}$ & $A_{3}$ & $A_{4}$ & $A_{5}$ \\
\cline { 2 - 7 }$T$ & $A_{6}$ & $A_{7}$ & $A_{8}$ & $A_{9}$ & $X$ \\
\cline { 2 - 7 }$T^{2}$ & $A_{10}$ & $A_{11}$ & $A_{12}$ & $A_{13}$ & $A_{14}$ \\
\hline $\mathrm{X}$ & $A_{15}$ & $X$ & $X$ & $A_{16}$ \\
\hline
\end{tabular}

Other thermodynamic functions, derived from $F$, can be summarized in a similar fashion:

$$
\mathrm{S}=-\mathrm{F}_{\mathrm{T}}
$$

\begin{tabular}{|c|c|c|c|c|c|}
\hline & 1 & م & $\rho^{2}$ & $\rho^{3}$ & $\ln p$ \\
\hline $1+\ln T$ & $-A_{6}$ & $-A_{7}$ & $-A_{8}$ & $-A_{9}$ & $X$ \\
\hline $\mathrm{T}$ & $-2 A_{10}$ & $-2 A_{11}$ & $-2 A_{12}$ & $-2 A_{13}$ & $-2 A_{15}$ \\
\hline 1 & $\mathrm{x}$ & $-A_{15}$ & $\mathrm{x}$ & $\mathrm{X}$ & $-A_{16}$ \\
\hline
\end{tabular}

$$
P=-F_{v}=\rho^{2} F_{\rho}
$$

\begin{tabular}{|c|c|c|c|c|}
\multicolumn{1}{c}{1} & $\rho^{2}$ & \multicolumn{2}{c}{$\rho^{3}$} & \multicolumn{2}{c}{$\rho^{A}$} & $\rho$ \\
\cline { 2 - 5 } & $A_{2}$ & $2 A_{3}$ & $3 A_{4}$ & $A_{5}$ \\
\cline { 2 - 5 }$T$ & $A_{7}$ & $2 A_{8}$ & $3 A_{9}$ & $X$ \\
\hline \multirow{2}{*}{$T$} & $A_{11}$ & $2 A_{12}$ & $3 A_{13}$ & $A_{14}$ \\
\hline$A_{15}$ & $X$ & $X$ & $A_{16}$ \\
\hline
\end{tabular}


$\varepsilon=\mathrm{F}-\mathrm{TF}_{\mathrm{T}}$

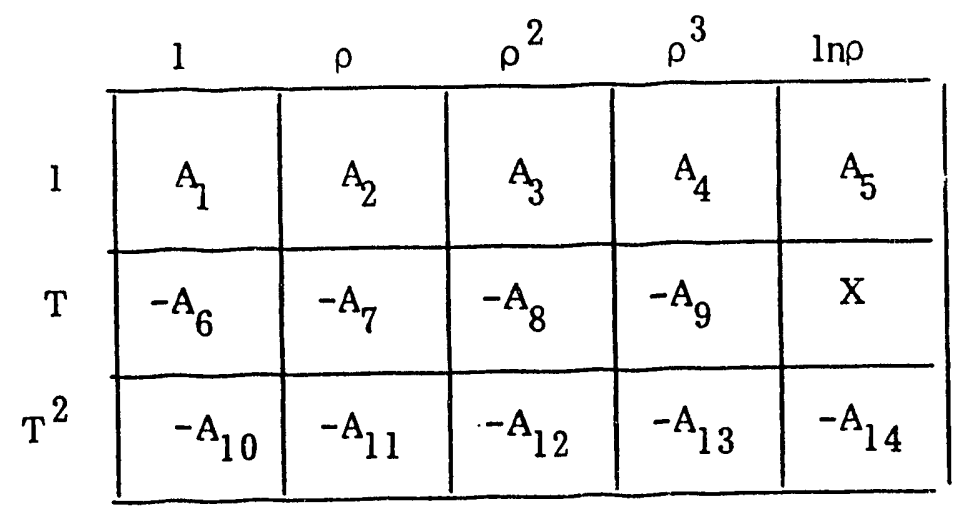

$\mathrm{P}_{\rho}=2 \rho \mathrm{F}_{\rho}+\rho^{2} \mathrm{~F}_{\rho \rho}$

\begin{tabular}{|c|c|c|c|c|}
\hline & $\rho$ & $\rho^{2}$ & $\rho^{3}$ & 1 \\
\hline 1 & $2 A_{2}$ & $6 \mathrm{~A}_{3}$ & $12 \mathrm{~A}_{4}$ & $A_{5}$ \\
\hline$T \ln T$ & $2 A_{7}$ & $6 A_{8}$ & $12 \mathrm{~A}_{9}$ & $\mathrm{x}$ \\
\hline $\mathrm{T}^{2}$ & $2 \mathrm{~A}_{11}$ & $6 \mathrm{~A}_{12}$ & $12 \mathrm{~A}_{13}$ & $\mathrm{~A}_{14}$ \\
\hline$T$ & $2 \mathrm{~A}_{15}$ & $x$ & $x$ & $A_{16}$ \\
\hline
\end{tabular}

$\mathrm{C}_{\mathrm{v}}=\varepsilon_{\mathrm{T}}=-\mathrm{TF}_{\mathrm{TT}}$

\begin{tabular}{|c|c|c|c|c|}
\hline 1 & $\rho$ & $\rho^{2}$ & $\rho^{3}$ & $\ln p$ \\
\hline$-A_{6}$ & $-A_{7}$ & $-A_{8}$ & $-A_{9}$ & $\mathrm{X}$ \\
\hline$-2 \mathrm{~A}_{10}$ & $-2 A_{11}$ & $-2 \mathrm{~A}_{12}$ & $-2 \mathrm{~A}_{13}$ & $-2 \mathrm{~A}_{14}$ \\
\hline
\end{tabular}




$\mathrm{P}+\varepsilon_{\mathrm{V}}=\mathrm{TP}_{\mathrm{T}}=\mathrm{T}^{2} \mathrm{~F}_{\rho \mathrm{T}}$
\begin{tabular}{r|c|c|c|c|}
$\rho^{2}$ & $\rho^{3}$ & $\rho^{4}$ & $\rho$ \\
\hline $\mathrm{T}(1+\ln \mathrm{T})$ & $\mathrm{A}_{7}$ & $2 \mathrm{~A}_{8}$ & $3 \mathrm{~A}_{9}$ & $\mathrm{X}$ \\
\cline { 2 - 5 } $\mathrm{T}^{2}$ & $2 \mathrm{~A}_{11}$ & $4 \mathrm{~A}_{12}$ & $6 \mathrm{~A}_{13}$ & $2 \mathrm{~A}_{14}$ \\
\cline { 2 - 5 } $\mathrm{T}$ & $\mathrm{A}_{15}$ & $\mathrm{X}$ & $\mathrm{X}$ & $\mathrm{A}_{16}$ \\
\hline
\end{tabular}

It should be noted that the fundamental idea of the FEEST treatment of equations of state is that $\mathrm{F}$ is represented by an expression linear in a set of chosen basis functions, $f_{n}(T, 0)$ and any required thermodynamic functions are obtained by taking appropriate combinations of the derivatives of $F$. It is a trivial matter to modify the fitting code (MEFISTO, see FITTING CODES) to embrace any oci-r: set of basis functions. The basis set presented abcve has been selected by heuristic argument and experimentation in fitting actual equation of state data.

Furthermore, the concept of the FEEST system can be extended to the treatment of independent variables other than $T$ and $\rho$. For example, one may choose $S$ and $\rho$ as independent variables and tabulate internal energy $a(S, \rho)$ as the thermodynamic potential. The selection of $T$ and $P$ as independent variables would imply the tabulation of the Gibbs Free Energy, $G(T, P)=\varepsilon-T S+P v$ and selection of $S$ and $P$ as independent variables implies tabulation of the enthalpy $H(S, P)=\varepsilon+P v$. In any of these cases, the required modifications in MEFISTO, the equation of state fitting code, are trivial.

We have chosen to tabulate $F(T, \rho)$ only because $T$ and $\rho$ are traditionally the choice of independent variables in computer calculations involving both hydrodynamics and radiation transport. Many LLNL computer codes have been tied to this choice of independent variables. Another interesting possibility is to treat entropy as a thermodynamic potential. One could tabulate $S$ as a function of $\varepsilon$ and $v$ (or $\rho$ ) and derive $T$ from $T=1 /\left(\frac{\partial S}{\partial \varepsilon}\right)_{v}$ and $P$ from $P / T=(\partial S / \partial v)_{\varepsilon}$. 


\section{Phase Changes}

Note: Neither the bi-quadratic nor the FEEST systems are designed to treat phase changes. Both tabulation schemes presume the tabulated function and (at least) its first derivations are continuous within a table box (or region).

Phase change lines seldom coincide with isotherms or isochors. Rather, they cut diagonally across table boxes. Attempts to fence the biquadratic tables to fit multi-phase equations of state have been the source of much grief - and large inaccurate tables.

It is strongly suggested that various phases be fit with separate tables and that the boundaries of phase regions be defined through a separate tabulation, e.g., $T_{\text {p.c. }}(\rho)$ where $T_{\text {p.c. }}$ is the temperature where the phase change occurs and hence where one must switch from the EOS tables appropria te for one phase to thos's appropriate for another phase.

An alternate approach which may be worth exploration is to tabulate the Gibbs free energy $G(P, T)$. Box boundaries would fall on line $P=$ constant, and could be selected to correspond with the phase change lines - at least for first-order phase transitions.

\section{Opacity}

The opacity is represented by

$$
\ln K=\sum B(M, n) K_{n}(T, \rho)
$$

The basis set, $\mathrm{K}_{\mathrm{n}}$, which has been selected is

$$
\begin{aligned}
\mathrm{K}_{1} & =1 \\
\mathrm{~K}_{2} & =\ln \rho \\
\mathrm{K}_{3} & =(\ln \rho)^{2} \\
\mathrm{~K}_{4} & =\ln \mathrm{T} \\
\mathrm{K}_{5} & =(\ln \rho)(\ln \mathrm{T}) \\
\mathrm{K}_{6} & =(\ln \rho)^{2} \ln \mathrm{T} \\
\mathrm{K}_{7} & =(\ln \mathrm{T})^{2} \\
\mathrm{~K}_{8} & =(\ln \mathrm{T})^{2} \ln \rho \\
\mathrm{K}_{9} & =(\ln \mathrm{T})^{2}(\ln \rho)^{2}
\end{aligned}
$$




\section{FITTING CODES}

Two computer programs are used to produce the tabular fits to equation of state and opacity data: MEFISTO produces a tabulation of coefficients for computing the Hemlholtz free energy, LUCIFER tabulates the coefficients for computing opacities. The method for fitting is essentially the same in both MEFISTO and LUCIFER, but LUCIFER is somewhat simpler and faster since it must fit only opacity data and determines only 9 coefficients per region whereas MEF ISTO may take as input $F, P, \varepsilon, \varepsilon_{\mathrm{T}}$, $\mathrm{P}_{\rho}, \varepsilon_{\mathrm{v}}, \mathrm{P}_{\mathrm{T}}$, or $\mathrm{S}$ data or any combination of these and must determine 16 coefficients per region.

We will not describe either of these codes in detail here; their structure and use will be the subject of a separate report. We will, hcwever, outline the method, taking MEFSTO as the model.

The $T-\rho$ plane is divided into regions composed of adjoint or disjoint rectangles bounded by lines $T=$ constant, $\rho=$ constant. Each region is assigned a number. We wish to find a set of coefficients $A(M, n)$ for each region, $M$, such that the expression

$$
F=\sum_{n} A(M, n) f_{n}(T, \rho)
$$

provides a fit to the various data points falling within $M$. Furthermore, it is required that $\mathrm{F}$ and specified derivatives of $\mathrm{F}$ be continuous, or nearly continuous, across region intersection lines.

Each type of input data $\left(P, \varepsilon, \varepsilon_{T}\right.$, etc.) is associated with a particular function linear in the $A(M, n)$. For example

$$
\begin{aligned}
& P=\rho^{2} F_{\rho}=\sum_{n} \rho^{2} A(M, n) \frac{\partial f_{n}}{\partial \rho} \\
& \varepsilon=F-T F_{T}=\sum_{n} A(M, n)\left(f_{n}-T \frac{\partial f_{n}}{\partial T}\right), \text { etc. }
\end{aligned}
$$

Denote by $F^{\alpha}(T, \rho)$, the expression associated with data type $\alpha$ and by $f_{n}^{\alpha}$ the corresponding basis functions (e.g., for $P, F^{l}=\rho^{2} F_{\rho}$ and $f_{n}^{l}=\rho^{2} \frac{\partial f n}{\partial \rho}$, etc.). For each data point $\mathrm{F}_{\mathrm{i}}^{\alpha}\left(\mathrm{T}_{\mathrm{i}}, \mathrm{O}_{\mathrm{i}}\right)$ there is a corresponding expression to approximate the data:

$$
F^{\alpha}\left(T_{i}, \rho_{i}\right)=\sum_{n} A(M, n) f_{n}^{\alpha}\left(T_{i}, \rho_{i}\right)
$$


The relative error (residual) for this point is

$$
R_{i}^{\alpha}=\left[F_{i}^{\alpha}-F^{\alpha}\left(T_{i}, \rho_{i}\right)\right] / F_{i}^{\alpha}
$$

If we ignore the fact that $\mathrm{F}$ must be continuous across region boundaries, then the $A(M, n)$ are determined by the least squares criterion: they are selected so that

$$
\sum_{\alpha} \sum_{i}\left(W_{i} R_{i}^{\alpha}\right)^{2} \text { is minimal ; }
$$

where $W_{i}$ is the weight (usually unity) associated with the data point $F_{i}^{\alpha}\left(T_{i}, \rho_{i}\right)$.

Continuity across region boundaries is not strictly enforced. (Indeed, for some combinations of region shapes the coupling required by strict continuity could overdetermine the $A(M, n)$ 's and hence continuity cannot be strictly enforced.) Instead, continuity is required only in a least squares sense.

Let $\mathrm{F}_{\mathrm{b}}^{\beta+}$ be the value of $\mathrm{F}^{\beta}$ computed from Eq. (10) using coefficients for the region on one side of the region boundary at the point $\left(T_{b}, q_{b}\right)$ and $F_{b}^{\beta-}$ be the value computed using coefficients for the region on the other side. A discrete set of points $\left(T_{b}, p_{b}\right)$ (10 along each box boundary) is generated, and a residual is computed for each of these points:

$$
\mathrm{R}_{\mathrm{b}}^{\beta}=\left(\mathrm{F}_{\mathrm{b}}^{\beta+}-\mathrm{F}_{\mathrm{b}}^{\beta-}\right) / \tilde{F}_{b}^{\beta}\left(\mathrm{T}_{b^{,}, \rho_{b}}\right) \text {, }
$$

where $\widetilde{F}_{b}$ is a guess at the value of $F^{\beta}$ at $\left(T_{b}, q_{b}\right)$ (the "guess" is improved by iteration as the codt converges on the "best" fit). The sum of the squares of these residuals (appropriately weighted) is added to the squared residual of Eq. (11) and the fit is then the choice of the $A(M, n)$ for which

$$
\sum_{\alpha} \sum_{i}\left(W_{i} R_{1}^{\alpha}\right)^{2}+\sum_{\beta} \sum_{b}\left(W_{b} R_{b}^{\beta}\right)^{2} \text { is minimal }
$$

MEFISTO attempts to achieve continuity of $F, P, \varepsilon, P_{\rho}$, and $\varepsilon_{T}$ (i.e., essentially, $F$ and its first and second derivatives) across region boundaries. The weights $W_{b}$ are set so that $\mathrm{F}, \mathrm{P}$, and $\varepsilon$ are continuous to $<0.5 \%$ and $\mathrm{P}_{\rho}$ and $\varepsilon_{\mathrm{T}}$ are continuous to $<20 \%$ (with but a few exceptions). 
LUCIFER attempts to achieve continuity only in $\kappa$ and the weights $w_{b}$ are set so that $K$ is continuous to $<0.5 \%$.

A third code: MEFTV produces an analysis of the fits produced by MEFISTO and LUCIFER. MEFTV provides a map of the fitting errors and plots of the fits.

Output from MEFSTO and LUCIFER includes: (1) a table file (MEFTAB); (2) an input file for MEFTV (MTVIN); and (3) a printed output file (MEFOUT) including the table grid, region map, a listing of all data points and the fitting error for each point, and an analysis of discontinuity at region intersection lines. 

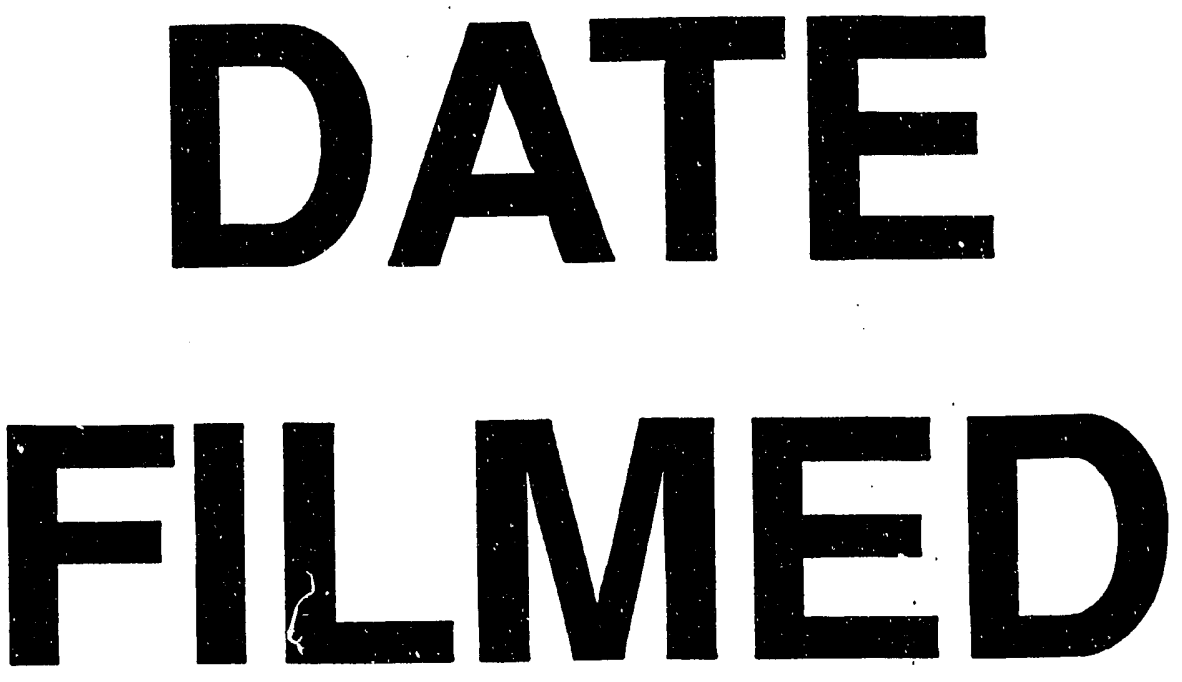

$9 / 10 / 93$
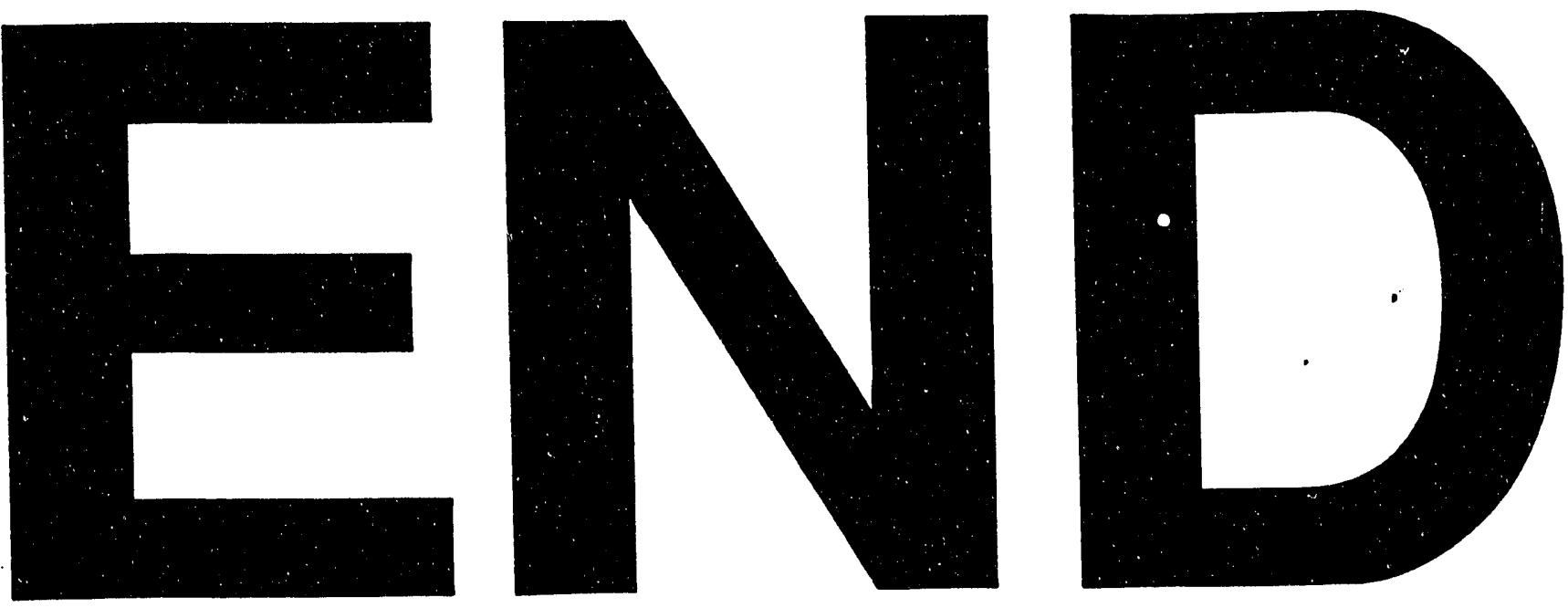
